\title{
Variable Radio Emission from X-Ray Sources
}

\author{
L. L. E. BRAES and G. K. MILEY (Leiden)
}

Dr. KELLOGG has just described some exciting new observations of $\mathrm{X}$-ray sources made with the UHURU satellite. We shall now move some nine orders of magnitude in wavelength to the opposite end of the electromagnetic spectrum and report measurements of weak radio emission from some of the objects he mentioned. For the detection of weak sources most radio telescopes are not noise limited, but are confusion limited by their low resolution. The aperture synthesis technique minimizes this problem because it enables one to pinpoint the position of weak sources to the order of one second of arc.

During the past year we have been making observations of galactic X-ray sources with the new Westerbork Synthesis Radiotelescope at a frequency of $1415 \mathrm{MHz}$ (BRAES and BROUW, 1971; BRAES and MILEY, 1971a, b). The Westerbork instrument is an east-west array of twelve $25-\mathrm{m}$ dishes. Ten of these are fixed in position while the other two are movable along a rail track, giving a maximum interferometer baseline of $1.6 \mathrm{~km}$. A Fourier transform of the correlated data from 20 separate interferometers gives a radio map of a region of the sky $0.6^{\circ}$ to half power diameter, with an angular resolution of a few seconds of arc. The r. $\mathrm{m}$. s. noise level for one 12 hour observation is about 1,3 milliflux units *).

We observed a total of 11 objects and have definitely detected $1415 \mathrm{MHz}$ radiation from three of them - Scorpius $X-1, G X 17+2$ and Cygnus $X-1$. In every case $1415 \mathrm{MHz}$ is so far the lowest frequency at which radiation has been detected. All three radio identifications satisfy two criteria. Firstly, the radio source is of course within the $\mathrm{X}$-ray position errors and secondly the radio emission is strongly variable. The character of both the radio variations and the X-ray emission of Cygnus X-1 differs radically from that of Sco X-1 and GX $17+2$ suggesting that there are at least two classes of these radio emitting $X$-ray-sources. We shall therefore first discuss Sco $X-1$ and GX $17+2$ and later talk about our observations of Cygnus X-1.

\section{Scorpius $X-1$}

Figure $1 \mathrm{~b}$ shows a synthesis map produced from one 7-hour observation of the Sco X-1 field made on March 30,1971 . At the centre of this map is a triple radio source first reported by HJELLMING and WADE (1971a) at 2695 and $8085 \mathrm{MHz}$. The central component of this source coincides in position with the well known variable X-ray star. Figure 1 a shows a similar map observed on the previous day, and here the central component is virtually absent. In less than 24 hours it has increased in strength by a factor of five. As part of an international campaign we monitored the behaviour of the central component over 10 successive days. The results, shown in Figure 2, show no evidence of any regularity. Also, simultaneous five-colour photometry of the X-rax star by PEL at the Leiden Southern Station reveals no correlation between radio and optical variations. A comparison of our measurements with simultaneous observations at 2695 and $8085 \mathrm{MHz}$ (HJELLMING and WADE 1971c) suggest that the central component has a non-thermal spectrum which becomes optically thick between 2695 and $1415 \mathrm{MHz}$.

By contrast with the central component the outer components do not appear to vary significantly, each having a strength of about $20 \mathrm{~m}$. f. u. They also have non-thermal spectra with at least one becoming optically thick between 1415 and $408 \mathrm{MHz}$. The two of them are located quite symmetrically $\sim 1.5$ from the $\mathrm{X}$-ray star and it seems reasonable to assume that they are relativistic plasmoids that were ejected from this star in a previous outburst. No conclusive evidence has yet been found for proper motion.

*) 1 m.f.u. $=10^{-29} \mathrm{~W} \mathrm{~m}^{-2} \mathrm{~Hz}^{-1}$ th $\mathrm{r}^{--1}$. 


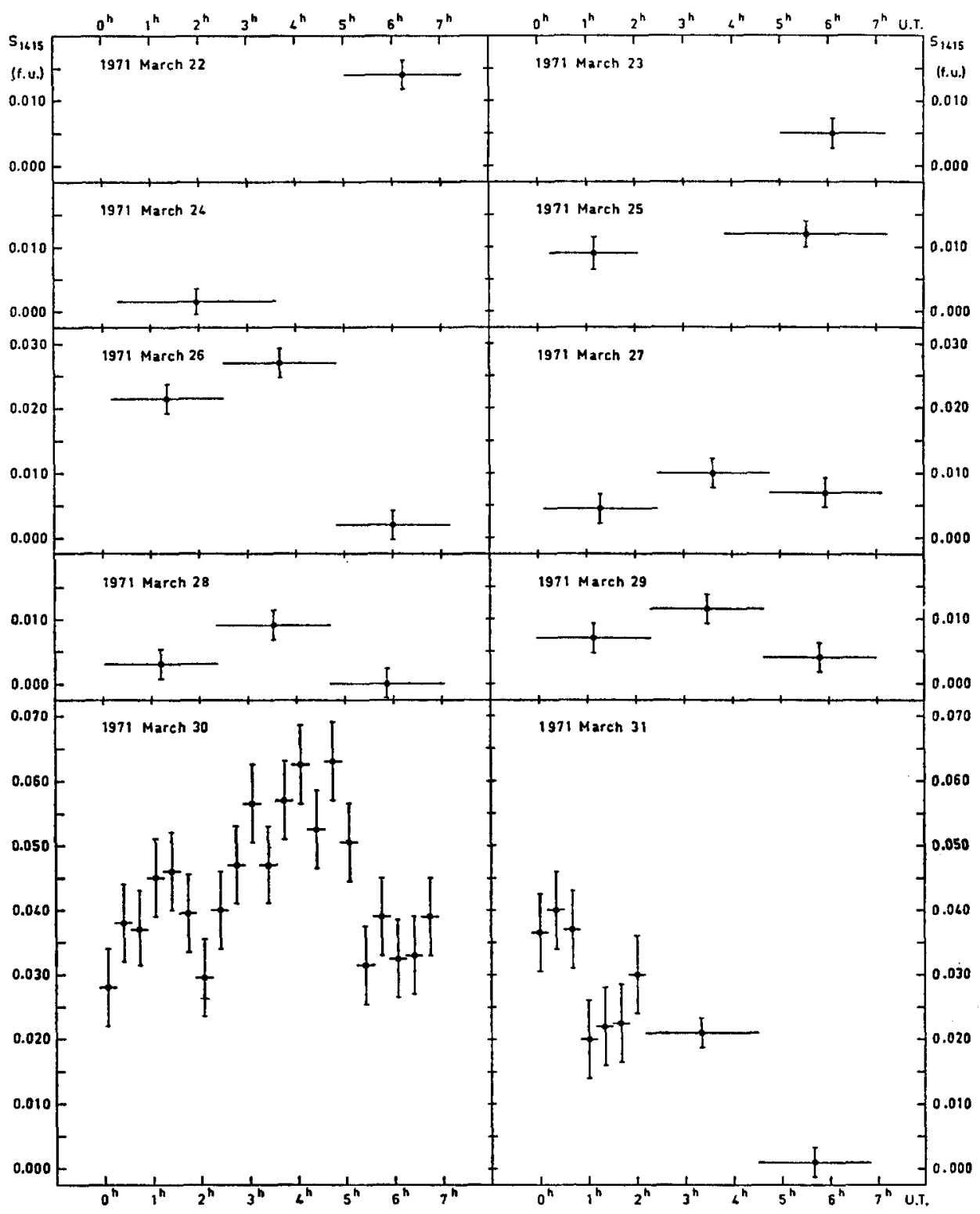

Fig. 2: $1415 \mathrm{MHz}$ brightness of the central component of Sco X-1 as a function of time on ten successive days. Each point represents an average over either 20 or 140 minutes depending on the strength of the source, and the vertical bars indicate mean errors. 


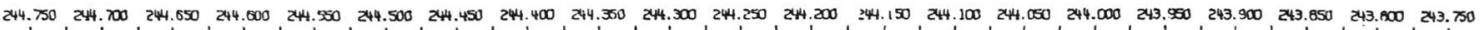

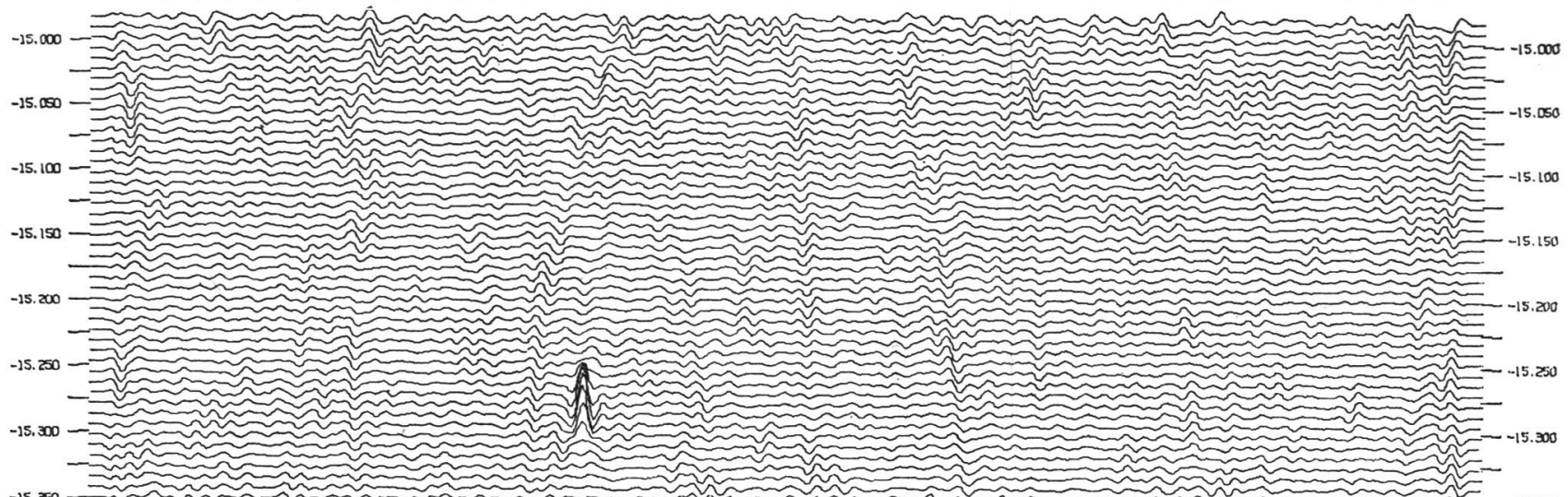

多.
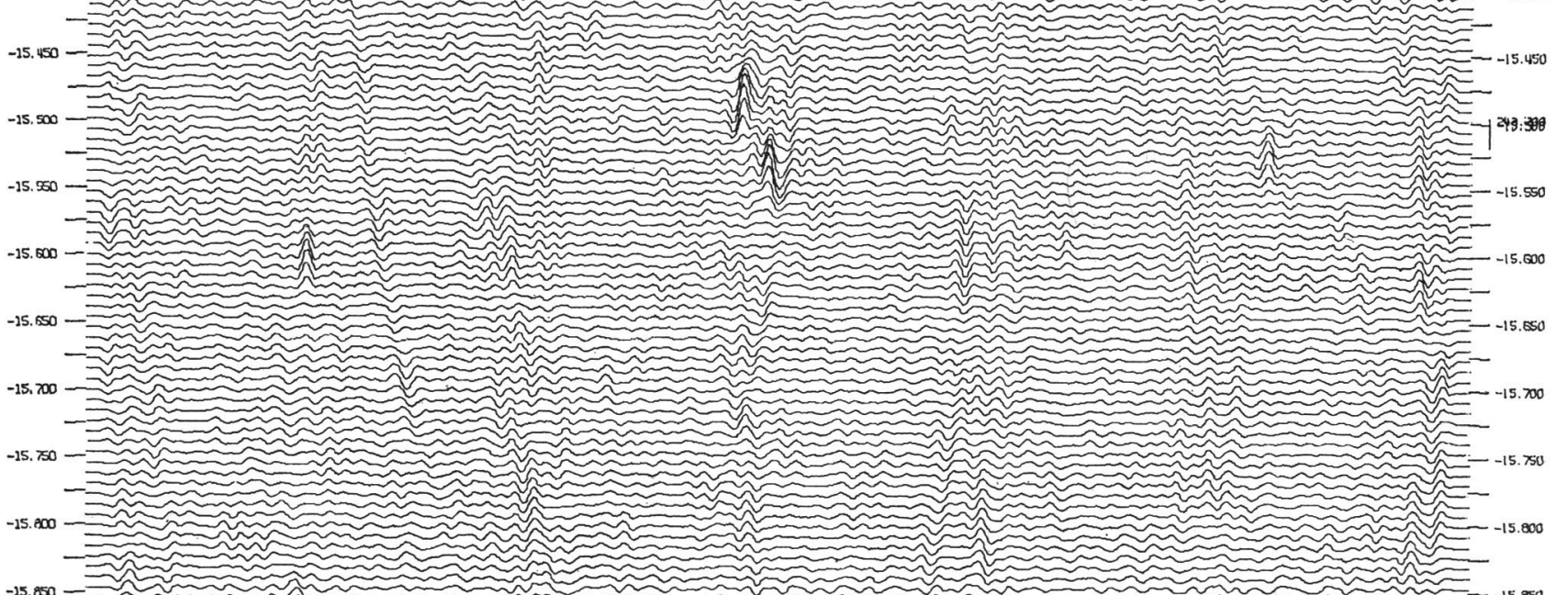

15.850,

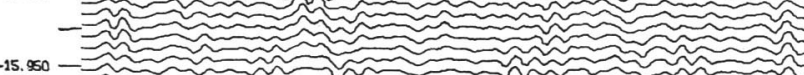

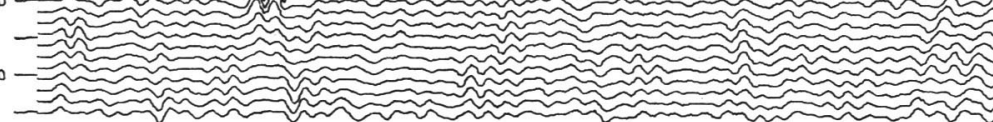

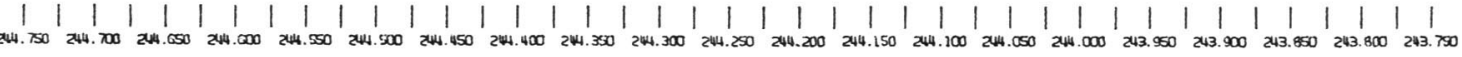

Fig. 1a: $1415 \mathrm{MHz}$ map of a $1^{\circ} \times 1^{\circ}$ region centered on Sco X-1 made on March 29. The abscissae and ordinates are 1950 right ascension at declination labelled in degrees.
100032905 Published online by cambridge University press 


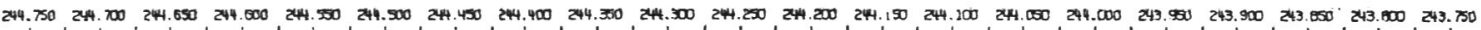

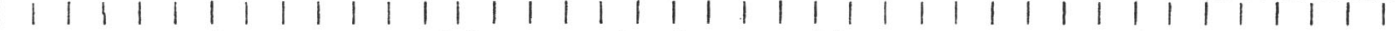

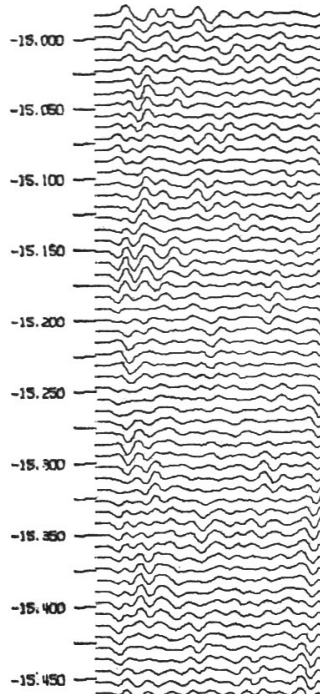

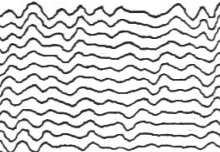
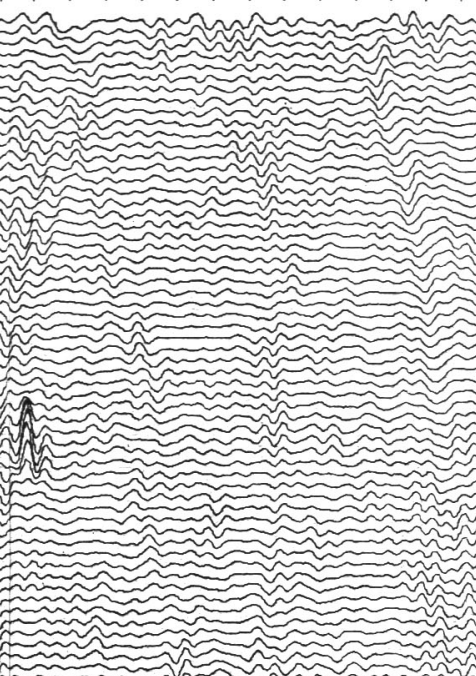

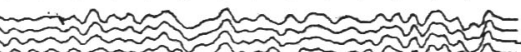
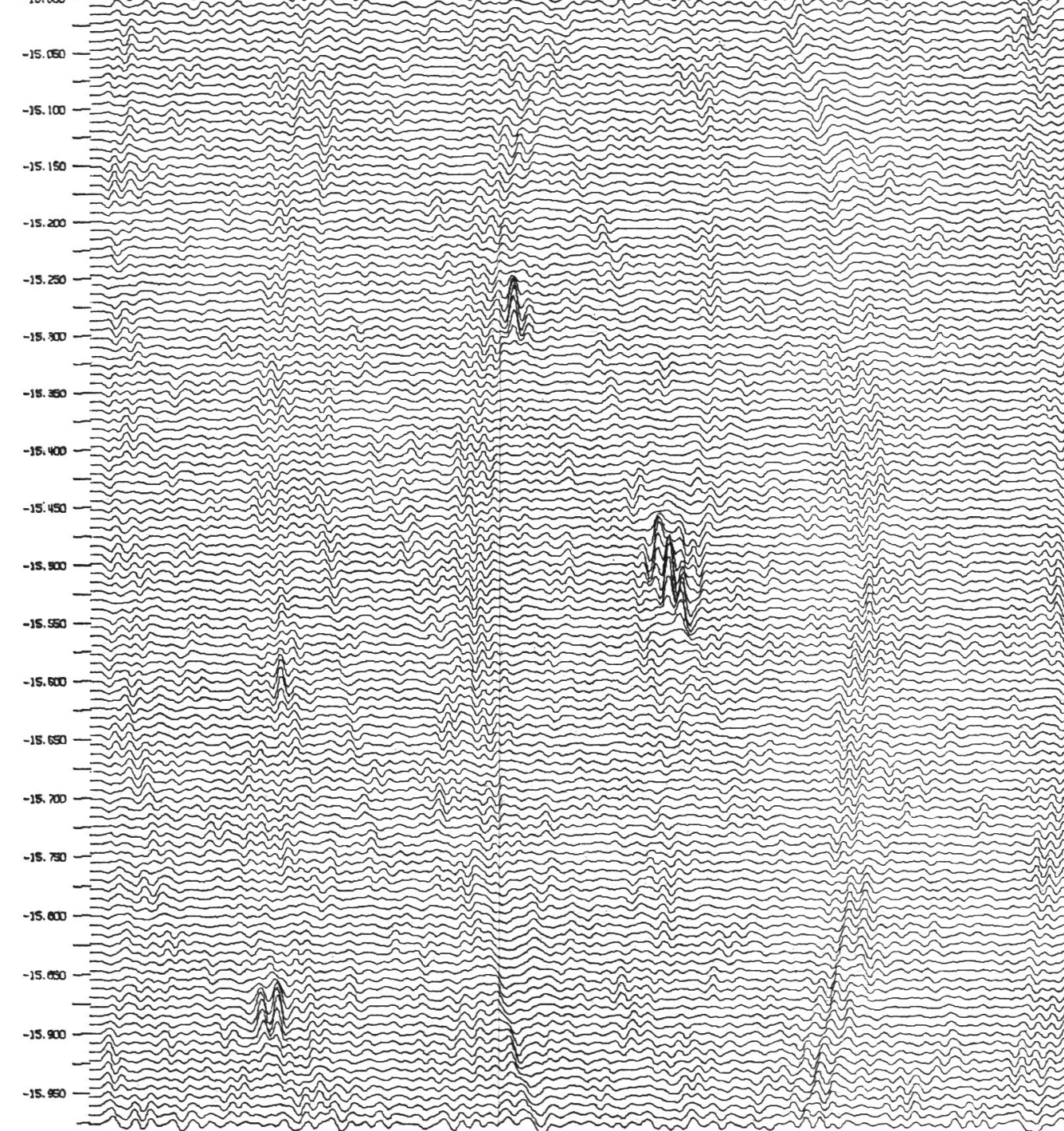

I 1

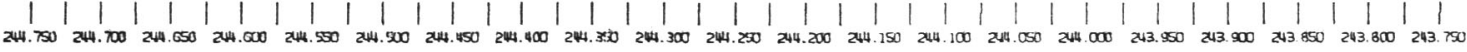

Fig. 1b: $1415 \mathrm{MHz}$ map of a $1^{\circ} \times 1^{\circ}$ region centered on Sco X-1 made on March 30. The

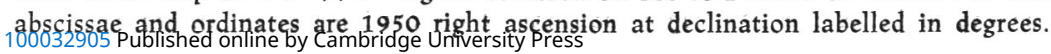


We have detected a point radio source with a flux density of $12 \mathrm{~m}$. f. $\mathrm{u}$. within the X-ray error box of GX17+2( $\left.\sim 2^{\prime} \times 1^{\prime}\right)$. The same source has been detected at 2695 and $8085 \mathrm{MHz}$ by HJELLMING and WADE (1971b) who report that it varies erratically in a similar manner as Sco X-1. From a comparison with their data the source appears to have a flat radio spectrum. An optical identification cannot yet be given.

\title{
Cygnus $X-1$
}

Like Sco X-1 and GX $17+2$, the radio source detected at the X-ray position of Cyg X-1 is also highly variable, but in a very different way. Between June 14, 1970, and March 22, 1971, it has been observed three times by HJELLMING and WADE, and once by us. On no occasion was any source detected above $5 \mathrm{~m}$. f. u. On April 28, 1971, we found a source at the X-ray position with a $1415 \mathrm{MHz}$ flux density of $21 \mathrm{~m}$. f. u. HJELLMING and WADE (1971b) detected it at $2695 \mathrm{MHz}$ on May 13. After that they observed it on nine occasions but saw no short term variability. Thus it would appear that some time between March 22 and April 28 the source increased in brightness by more than a factor of four and remained constant afterwards.

The radio emission is within $1^{\prime \prime}$ of the 9th magnitude Bo lb star HD 226868 . The probability of a chance coincidence with such a rare type of star is negligible and therefore we feel that the radio source is in some way associated with it. The character of the radio variations would appear to support DOLAN's suggestion that Cygnus X-1 is an eclipsing binary system (DOLAN, 1970, BIDELMAN and WEITENBECK, 1971) but continued monitoring of the radio emission is needed to confirm this.

The Westerbork Radio Observatory is operated by the Netherlands Foundation for Radio Astronomy with the financial support of the Netherlands Organization for the Advancement of Pure Research (Z.W.O.).

\author{
References:
}

BIDELMAN, W. P., and WEITENBECK, A. J., 1971, IAU Circ. No. 2345.

BRAES, L. L. E., and BROUW, W. N., 1971, Astron. Astrophys. 12, 320.

BRAES, L. L. E., and MILEY, G. K., 1971a, Nature, 232, 246.

BRAES, L. L. E., and MILEY, G. K., 1971b, Astron. Astrophys. 14, 160.

DOLAN, J. F., 1970, Space Sci. Rev., 10, 830.

H JELLMING, R. M., and WADE, C. M., 1971a, Astrophys. J. 164, L1.

H JELLMING, R. M., and WADE, C. M., 1971b, Astrophys. J. 168, L21.

H JELLMING, R. M., and WADE, C. M., 1971c, submitted to Astrophys. J.

\section{Discussion to the paper of BRAES and MILEY}

HUTCHINGS: Does the distribution of your observations definitely exclude the possibility of a short period eclipsing binary?

BRAES: Yes. Dr. HJELLMING and Dr. WADE observed the $C_{y g} X-1$ radio source on four successive days in May. They detected no variations.

KUNKEL: For what it is worth: we monitored a $15^{\text {th }}$ magnitude red star within a few arc seconds of the GX $17+2$ position by Dr. HJELLMING and Dr. WADE with the equip. ment described by Dr. LASKER and Dr. HESSER and found essentially no variability. It is probably a foreground object not associated with the radio source.

SCHWARTZ: Would you care to comment about not detecting $\mathrm{Cyg}_{\mathrm{X}} \mathrm{X}-1$ on 4 occasions and than seeing it. Do you believe those early observations?

BRAES: Yes. Three of those observations were by Dr. HJELLMING and Dr. WADE and one was ours, so they were independent. And, we both subsequently detected it independently.

KELLOGG: Sco X-1, GX $17+2$ and $\mathrm{Cyg}_{\mathrm{X}} \mathrm{X}-2$ appear to be the same class of object, in that there is a variable $X$-ray source $( \pm 40 \%)$, a highly variable blue star and a highly variable radio source; with the following exceptions.

1) No star for GX $17+2$, probably due to observation.

2) No radio source for $\mathrm{Cyg} X-2$.

WARNER: Cyg X-2 is much fainter optically than Sco X-1, so the radio emission may be too weak to detect. Thus I see no reason why $\mathrm{Cyg} X-2$ should be placed in a different class to Sco X-1. 